\title{
References and Bibliography
}

This book was written to be as self-contained as possible, but the following related references may be useful for the readers.

The application of group theory in quantum mechanics is described in the following classical literature:

1. H. Weyl, The Theory of Groups and Quantum Mechanics, Dover (1983).

2. E. P. Wigner, Group Theory and its Application to the Quantum Mechanics of Atomic Spectra, Academic Press (1959).

3. B. L. van der Wearden, Die Gruppen Theoretische Methode in der Quantum Mechanik, Springer (1983).

For the recent literature see

4. M. Hamermesh, Group Theory and its Application to Physical Problems, AddisonWesley (1962).

As for the references on the representation theory of rotation group

5. T. Yamanouchi, The Rotation Group and its Representations, Iwanami (1957), is an appropriate introductory textbook.

The exact treatment of the representations of proper Lorentz group is found in

6. M. A. Naimark, Linear Representations of the Lorentz Group, Pergamon Press (1964).

7. I. M. Gel'fand, R. A. Minlos and Z. Y. Shapiro, Representations of Rotation and Lorentz Groups and Their Applications, Pergamon Press (1963). 
I do not know of any good textbooks on the unitary representations of the Poincaré group. Wigner's laborious work

8. E. P. Wigner, On Unitary Representation of the Inhomogeneous Lorentz Group, Ann. Math., 40 (1939) 149 is a classical and basic paper. Related papers are, for example,

9. E. P. Wigner, Relativistische Wellengleichungen, Z. Physik, 124 (1947), 665.

10. V. Bargmann and E. P. Wigner, Group Theoretical Discussion of Relativistic Wave Equations, Pro. Natl. Acad. Sci. U.S., 34 (1948) 211 and this subject was reviewed in

11. Ju M. Shirokov, A Group-Theoretical Consideration of the Basis of Relativistic Quantum Mechanics I, II, III, IV, Soviet Phys. - JETP, 6 (1958) 664, 918, 929, ibid., 7 (1958) 493.

The irreducible decomposition of direct products of the Poincaré group and the kinematics of $S$-matrices which were not considered in the present book can be found in

12. H. Joos, Zur Darstellungstheorie der Inhomogenen Lorentzgrouppe als Grundlage Quantenmechanische Kinematick, Fortschritte der Phys., 10 (1962) 65.

Many old papers on relativistic wave equation are referred in

13. E. M. Corson, Introduction to Tensors, Spinors, and Relativistic Wave-Equations, Hafner (1953).

The basic considerations of field quantization and the general theory of its application to systems with interactions are written in

14. Y. Takahashi, An Introduction to Field Quantization, Pergamon Press (1968).

The examples of quantum fields applied to particle physics can be found in

15. K. Nishijima, Field and Particles, Benjamin (1969).

16. N. Nakanishi, Quantum Field Theory, Baifukan (1975).

17. H. Yukawa and Y. Katayama, Elementary Particle Theory (Iwanami lectures of the foundation of modern physics 11), Iwanami (1974). 


\section{Index}

Angular momentum 63, 72

Angular momentum density 200

Annihilation operator 175

Anti-particle 190

Anti-unitary 3, 114, 203

Bargmann-Wigner amplitude 95

Bargmann-Wigner equation 95, 143

Bose statistics 181, 183, 199

Casimir operator 25

Charge conjugation 117, 165, 169

Charge conjugation matrix 108

Complex conjugate representation 20

Complex scalar field 176

Conjugate representation 20

Continuous representation 55

Continuous spin 46, 156

Covariant formalism 81

Covariant inner product 83,151

Creation operator 175

Current density 191

Current operator 196

Diagonal 32

Diagonal $\gamma_{5} \quad 110$

Dirac equation 87,140
Dirac particle 85

Dirac representation 109

Dirac spinor 109

Discrete representation 56

Discrete spin 44, 135

Discrete transformation 112

Double-valued representation 14, 166

Dual space 203

E(2) 43

Energy density 174

Energy-momentum tensor 186, 198

Fermi statistics $178,183,199$

Field 171

Fiertz-Pauli equation 129

Foldy transformation 87

Free field 172, 191

Free particle 6, 57

Furry's theorem 111

GL(p) 101

$\gamma$ matrices 106

Gauge transformation 151

General linear transformation group 101

Generator 22 
Harmonic oscillator 176

Heisenberg equation of motion 173

Helicity 79

Higher spin 91

Hole theory 57

Identity transformation 3

Imaginary mass 58,70

Infinitesimal Lorents transformation 22

Infinitesimal rotation 21

Infinitesimal transformation 20

Irreducible representation 7

Kemmer type equation 84

Klein-Gordon equation 83

Left-handed polarization 146

Light-like 27

Little group 33

Locality condition 174

Lorents group 5, 47

Lorents transformation 5,31

Majorana field 208

Majorana representation 110

Matter wave 172

Momentum density 200

Natural unit 5

Negative frequency $\mathbf{5 8}$

Norm 1

Null vector 27

Number operator 195

Orbital angular momentum 63

Para-Bose statistics 184, 191, 208

Para-Fermi statistics 184, 208

Parameter space 8

Pauli matrices 14

Poincare' group 6

Polarisation 79

Position operator 85

Positive frequency 58

Principal series 51
Proca equation 120

Rarita-Schwinger equation 124, 134

Real scalar field 176

Representation 3

Right-handed polarisation 146

Rotation axis 11

Rotation group 39

cr-surface 188

SL $(2, C) \quad 19$

SU(2) 20

Scalar field 184

Schur's lemma 19

Simply connected 9

Single-valued representation 14, 156

Space reflection 113, 144, 165, 169, 203

Space-like 28

Space-like surface 188

Spatial rotation 10

Spin 40

- 082

$-1 / 2140$

- 1 119, 148

$-3 / 2121,151$

$-\mathrm{n} / 2143$

-n 128

$-n+1 / 2 \quad 132$

Spin angular momentum 63

Spin freedom 28

Spin vector 40

Spinor representation 14,19

Spurion 58

Standard Young tableau 100

Superposition principle 1

Supplementary series 51

Tachyon 58

r-transformation 61

$\wedge$-transformation 61

3-dimensional Lorents group 52

Time reversal 114, 146, 165, 169, 203

Time-like 27

Trace 14

Translation 26 